\title{
Coordinated Spatial Pattern Formation in Biomolecular Communication Networks
}

\author{
Yutaka Hori, Hiroki Miyazako, Soichiro Kumagai, and Shinji Hara
}

\begin{abstract}
This paper proposes a control theoretic framework to model and analyze the self-organized pattern formation of molecular concentrations in biomolecular communication networks, emerging applications in synthetic biology. In biomolecular communication networks, bio-nanomachines, or biological cells, communicate with each other using a cell-to-cell communication mechanism mediated by a diffusible signaling molecule, thereby the dynamics of molecular concentrations are approximately modeled as a reaction-diffusion system with a single diffuser. We first introduce a feedback model representation of the reaction-diffusion system and provide a systematic local stability/instability analysis tool using the root locus of the feedback system. The instability analysis then allows us to analytically derive the conditions for the self-organized spatial pattern formation, or Turing pattern formation, of the bio-nanomachines. We propose a novel synthetic biocircuit motif called activator-repressor-diffuser system and show that it is one of the minimum biomolecular circuits that admit self-organized patterns over cell population.
\end{abstract}

प

\section{Introduction}

Designing the coordinated dynamical behavior of cell populations is one of the important challenges in synthetic biology. The study of cell population control is expected to provide not only the better understanding of biology but also the ability to build biomimetic nanomachines, or bio-nanomachines, for bioengineering applications such as tissue engineering and targeted drug delivery [1,2. In the last decade, researchers designed a variety of biochemical circuits that perform population-level behavior such as synchronized oscillations [3], population control [4,5], and multicellular computation 6 8. In these works, intercellular

Y. Hori is with Department of Computing and Mathematical Sciences, California Institute of Technology, CA 91125 USA. yhori@caltech.edu.H. Miyazako, S. Kuamgai and S. Hara are with Department of Information Science and Technology, The University of Tokyo, Tokyo 113-8656 Japan. \{Hiroki_Miyazako, Shinji_Hara\}@ipc.i.u-tokyo.ac.jp

Y. Hori was supported by JSPS Fellowship for Research Abroad. This work was supported, in part, by Grant-in-Aid for JSPS Fellows grant number 15J09841. 
coordination was enabled by a cell-to-cell communication mechanism called quorum sensing, where cells communicate with each other by releasing and receiving diffusible signaling molecules.

One of the earliest synthetic biocircuits for spatial pattern formation of cell population was demonstrated in Basu et al. 9, where sender cells transmit a quorum sensing autoinducer molecule and receiver cells respond by expressing fluorescent protein to form predefined spatial patterns. This work was later followed by Liu et al. [10], where another biochemical circuit was developed to form a periodic pattern of cell density in a self-organized manner. From an engineering point of view, self-organized pattern formation is preferable since it does not require extra design steps of external inputs and spatial allocation of cells. Moreover, the study of self-organization is important in developmental biology to understand morphogenesis [1, 12.

Recently, Hsia et al. [13] introduced a dynamical model of biomolecular communication networks using reaction-diffusion equations, where diffusion-based cell-to-cell communication was modeled based on the Fick's laws of diffusion 14. It was then shown that a biochemical communication network can form a selforganized concentration gradient of chemical species over cell populations despite the averaging effect of molecular diffusion. Such self-organized spatiotemporal pattern is known as Turing pattern [15].

Although the theory of Turing pattern formation was extensively studied over the last 60 years 14, 16, many existing works of Turing pattern formation, which are often applied to developmental biology (see [17 for example), are not directly applicable to today's engineering applications in biomolecular communication networks because of the difference of the number of diffusible molecules. Specifically, in biomolecular communication networks, only a single molecular species, or a small signaling molecule, can diffuse between cells and most proteins and mRNAs are localized in a cell, while the classical works assume that all molecular species can diffuse in the medium. Hence, it is desirable to develop a novel theoretical framework that specifically targets the systematic modeling and analysis of the spatio-temporal dynamics of biomolecular communication networks with a single diffusible molecule.

In this paper, we consider a class of reaction-diffusion systems that approximately models biomolecular communication networks, emerging applications in synthetic biology. Our interest here is to study the structural properties of the systems that come from the physical constraints of biomolecular communication and utilize them to derive an analysis framework rather than to study general reaction-diffusion systems. Specifically, the goals of this paper are (i) to present a stability analysis tool for biomolecular communication network systems and (ii) to use it to study the structures of biocircuits that are required for Turing pattern formation over the population of cells. From a synthetic biology viewpoint, it is useful to characterize the required structures of biocircuits as it specifies the design space of possible combinations of genetic parts.

We first present that the local stability analysis of the reaction-diffusion systems boils down to a simple graphical test using the root locus method [18. 
We show that the root locus is characterized by the dynamics of biocircuits inside cells and the complete circuit including diffusers, which allows us to provide physical interpretations of our theoretical results. Using the stability/instability analysis tool, we study conditions for Turing pattern formation in synthetic biomolecular communication networks. In particular, we mathematically prove that the minimum biocircuit producing coordinated spatial patterns is composed of three genes, and moreover, certain activation-repression network patterns are necessary. As an example of the three-component biomolecular circuits, we propose activator-repressor-diffusor motif and show that a synthetic biocircuit with this motif can admit Turing instability using illustrative numerical simulations.

The organization of this paper is as follows. In Section 2, we provide a control theoretic modeling framework for biomolecular communication networks and introduce a useful decomposition that simplifies the stability analysis of the system. Then, in Section 3 , we provide a systematic stability analysis tool based on the root locus method and demonstrate on a novel activator-repressor-diffusor biomolecular circuit. Section 4 is devoted to deriving the condition for Turing pattern formation. Then, in Section 5 , the class of reaction networks that can admit Turing patterns is characterized. Finally, Section 6 concludes the paper.

The authors' conference papers [19 20] illustrated the outlines of the Turing instability analysis method shown in this paper, and the proofs were partly presented in the paper written in Japanese 21]. In contrast with these papers, the present paper emphasizes emerging applications in synthetic biology. For this purpose, all results are presented in a two dimensional spatial domain. In particular, a novel activator-repressor-diffuser biomolecular circuit motif is proposed as an example of minimum biocircuits to achieve spatially coordinated pattern formation. Biological relevance and challenges for implementation are also discussed. In addition, we provide a formal mathematical statement of our key result, a necessary and sufficient exponential stability condition (Proposition 1 ), for the first time with a complete proof, then the relation with the existing theoretical result 22 is discussed. Other mathematical results are also shown with an updated rigorous assumption (Assumption 1) and complete proofs.

The following notations are used throughout this paper: $\mathbb{N}_{0}:=\{0,1,2, \cdots\}$. $\mathbb{R}_{0+}:=\{r \in \mathbb{R} \mid r \geq 0\} . \mathbb{C}_{+}:=\{c \in \mathbb{C} \mid \operatorname{Re}[c]>0\} . \mathbb{C}_{0+}:=\{c \in \mathbb{C} \mid \operatorname{Re}[c] \geq 0\}$. $\mathbb{C}_{-}:=\{c \in \mathbb{C} \mid \operatorname{Re}[c]<0\}$. $I_{n}$ is an $n$ by $n$ identity matrix.

\section{Modeling of Biomolecular Communication Net- works}

\subsection{Model description}

We consider molecular communication networks in a two dimensional medium $\Omega:=\Xi_{1} \times \Xi_{2}=\left[0, L_{1}\right] \times\left[0, L_{2}\right]$ where a large number of cells communicate using a single diffusible transmitter molecule as illustrated in Fig. 1. Each cell 


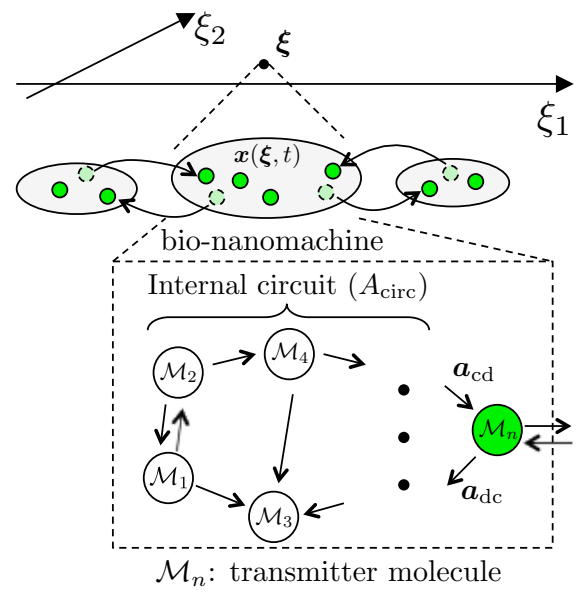

Figure 1: Molecular communication network. The chemical concentrations inside cells are approximately modeled by the continuous gradient of concentrations $\boldsymbol{x}(\boldsymbol{\xi}, t)$.

contains a genetically identical gene regulatory circuit that serves as a functional module such as bistable switch [23], oscillator [24], logic gates [25], and so on. The circuit is composed of $n-1$ molecular species $\mathcal{M}_{1}, \mathcal{M}_{2}, \cdots, \mathcal{M}_{n-1}$ and is called internal circuit (see Fig. 1). The molecules $\mathcal{M}_{1}, \mathcal{M}_{2}, \cdots, \mathcal{M}_{n-1}$ are, for example, transcription factors and associated mRNAs. In order for the cells to communicate, cells produce a transmitter molecule $\mathcal{M}_{n}$ and release it to the medium. An example of such transmitter molecule is $\mathrm{N}$-Acyl homoserine lactone (AHL), which is a small molecule that can diffuse through cell membrane and is used for quorum sensing of gram-negative bacteria. It should be noted that, in engineering applications, one could use engineered bio-nanomachines such as vesicles instead of living cells.

When the cells are densely populated in the medium, the chemical levels in the cells can be approximately modeled as the continuous gradient of the molecular concentrations inside cells. Let $x_{i}(\boldsymbol{\xi}, t)$ denote the concentration of $\mathcal{M}_{i}(i=1,2, \cdots, n)$ at position $\boldsymbol{\xi}:=\left[\xi_{1}, \xi_{2}\right]^{T} \in \Omega$ and at time $t$, and define $\boldsymbol{x}(\boldsymbol{\xi}, t):=\left[x_{1}(\boldsymbol{\xi}, t), x_{2}(\boldsymbol{\xi}, t), \cdots, x_{n}(\boldsymbol{\xi}, t)\right]^{T}$. The dynamics of the concentrations of $\mathcal{M}_{1}, \mathcal{M}_{2}, \cdots, \mathcal{M}_{n}$ are then modeled by

$$
\frac{\partial \boldsymbol{x}(\boldsymbol{\xi}, t)}{\partial t}=f(\boldsymbol{x}(\boldsymbol{\xi}, t))+D\left(\frac{\partial^{2} \boldsymbol{x}(\boldsymbol{\xi}, t)}{\partial \xi_{1}^{2}}+\frac{\partial^{2} \boldsymbol{x}(\boldsymbol{\xi}, t)}{\partial \xi_{2}^{2}}\right),
$$

where $f(\cdot)$ is a function representing the rate of production and degradation of the molecules. The matrix $D$ is defined as $D:=\operatorname{diag}(0, \cdots, 0, \mu) \in \mathbb{R}_{0+}^{n \times n}$ with a diffusion coefficient $\mu$. It should be noted that only the $(n, n)$-th entry is non-zero, since only the transmitter molecule $\mathcal{M}_{n}$ can diffuse between cells. 
We consider the Neumann boundary condition

$$
\overrightarrow{\boldsymbol{n}} \cdot \nabla \boldsymbol{x}(\boldsymbol{\xi}, t)=0 ; \quad \forall \boldsymbol{\xi} \in \partial \Omega,
$$

where $\overrightarrow{\boldsymbol{n}}$ is a vector normal to the boundary $\partial \Omega$. Throughout this paper, we assume that the model (1), the boundary condition and initial values are well-posed (see 26] and Chapter 14 of [27]) and that the Jacobian of $f(\cdot)$ is well-defined at $\boldsymbol{x}$ satisfying $f(\boldsymbol{x})=0$.

Let $\overline{\boldsymbol{x}} \in \mathbb{R}_{0+}^{n}$ denote an equilibrium state of a single cell, i.e., $f(\overline{\boldsymbol{x}})=0$. It follows that the right-hand side of (1) becomes zero when $\boldsymbol{x}(\boldsymbol{\xi}, t)=\overline{\boldsymbol{x}}$ for all $\xi \in \Omega$, because the concentration of each molecular species is uniform over the space and the spatial derivatives in (1) become zero. This implies that $\overline{\boldsymbol{x}}$ is a spatially homogeneous, or spatially uniform, equilibrium state of the system (1). It should be noted that the spatially homogeneous equilibrium state might not be unique. The local dynamics around $\overline{\boldsymbol{x}}$ are modeled by the following linearized model of (1).

$$
\frac{\partial \tilde{\boldsymbol{x}}(\boldsymbol{\xi}, t)}{\partial t}=A \tilde{\boldsymbol{x}}(\boldsymbol{\xi}, t)+D\left(\frac{\partial^{2} \tilde{\boldsymbol{x}}(\boldsymbol{\xi}, t)}{\partial \xi_{1}^{2}}+\frac{\partial^{2} \tilde{\boldsymbol{x}}(\boldsymbol{\xi}, t)}{\partial \xi_{2}^{2}}\right),
$$

where $A \in \mathbb{R}^{n \times n}$ is the Jacobian of $f(\cdot)$ at $\overline{\boldsymbol{x}}$, and $\tilde{\boldsymbol{x}}(\boldsymbol{\xi}, t)$ is defined by $\tilde{\boldsymbol{x}}(\boldsymbol{\xi}, t):=$ $\boldsymbol{x}(\boldsymbol{\xi}, t)-\overline{\boldsymbol{x}}$. Note that the system (3) is an infinite dimensional linear timeinvariant system.

In Section 3 , we provide a systematic analysis tool for studying stability of the equilibrium point $\overline{\boldsymbol{x}}$ using the linearized model (3). We then show that cells can form spatially inhomogeneous gradient of concentrations over the population when the system (3) is locally unstable and satisfies certain conditions.

\subsection{Control theoretic formulation of biomolecular commu- nication networks}

In this section, we introduce a control theoretic model representation of the biomolecular communication network system. We show that the infinite dimensional system (3) can be decomposed into an infinite number of finite dimensional single-input single-output (SISO) subsystems that account for the dynamics of individual spatial modes, which will be a key result in developing a systematic stability analysis tool in the next section.

The linearized model (3) can be expressed as

$$
\begin{aligned}
& \frac{\partial \tilde{\boldsymbol{x}}(\boldsymbol{\xi}, t)}{\partial t}=A \tilde{\boldsymbol{x}}(\boldsymbol{\xi}, t)+\boldsymbol{e}_{n} u(\boldsymbol{\xi}, t) \\
& y(\boldsymbol{\xi}, t)=\boldsymbol{e}_{n}^{T} \tilde{\boldsymbol{x}}(\boldsymbol{\xi}, t),
\end{aligned}
$$

where $u(\boldsymbol{\xi}, t):=\mu \nabla^{2} y(\boldsymbol{\xi}, t)$ with the Laplace operator

$$
\nabla^{2}:=\frac{\partial^{2}}{\partial \xi_{1}^{2}}+\frac{\partial^{2}}{\partial \xi_{2}^{2}}
$$




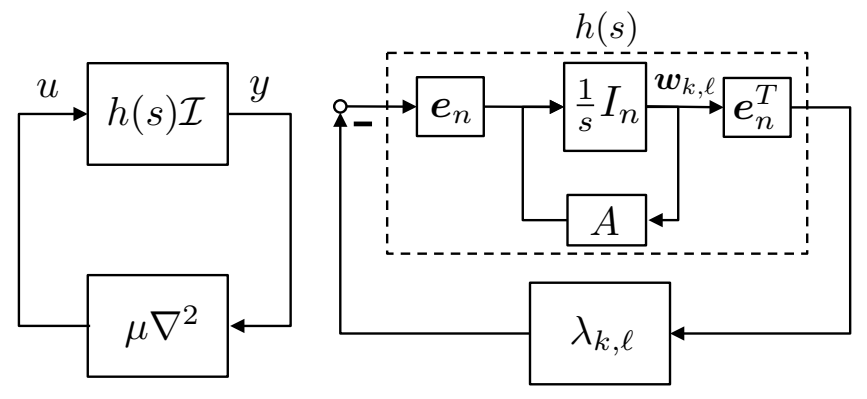

Figure 2: (Left) Feedback system representation of biomolecular communication network. (Right) Decomposed subsystem $\Sigma_{k, \ell}$.

and $\boldsymbol{e}_{n}:=[0, \cdots, 0,1]^{T} \in \mathbb{R}^{n}$. The equations (4) and (5) imply that for each fixed position $\boldsymbol{\xi}$, the dynamics of the local reactions inside a single cell can be modeled as a SISO system with the input $u(\boldsymbol{\xi}, t)$ and the output $y(\boldsymbol{\xi}, t)$. Note that $y(\boldsymbol{\xi}, t)$ is the concentration of the transmitter molecule $\mathcal{M}_{n}$ at position $\boldsymbol{\xi}$. For each $\boldsymbol{\xi}$, the transfer function from $u$ to $y$ is obtained as

$$
h(s):=\boldsymbol{e}_{n}^{T}\left(s I_{n}-A\right)^{-1} \boldsymbol{e}_{n}(=: n(s) / d(s)),
$$

where we define $n(s)$ and $d(s)$ as the numerator and the denominator of $h(s)$, respectively. As a result, the system can be illustrated as the feedback system shown in Fig. 2 (Left), where $\mathcal{I}$ is an identity operator. Note that the feedback system can be viewed as a multi-agent dynamical system, where the homogeneous agents $h(s)$ communicate with the nearest neighbors by the Laplace operator $\mu \nabla^{2}$. The stability analysis methods of a finite dimensional version of such systems were established in 28,29. Here we use the same approach to analyzing the stability of the infinite dimensional system (3).

Assumption 1. We assume $\left(A, \boldsymbol{e}_{n}\right)$ is stabilizable, $\left(A, \boldsymbol{e}_{n}^{T}\right)$ is detectable, and $h(s)$ does not have zeros on the imaginary axis, that is, $n(j \omega) \neq 0$ for all $\omega \in \mathbb{R}$.

Stabilizability and detectability guarantees the stability of the internal states, or molecular concentrations, that one cannot aware of and/or influence, if any. The readers are referred to Chapters 14 and 16 of 30 for the mathematical details. We also note that the non-existence of the zeros of $h(s)$ on the imaginary axis is not restrictive in many biological applications as shown after Lemma 1 in Section 3.

The stability analysis of the system (3) is not necessarily straightforward due to the infinite dimensionality of the system. In what follows, we show that the infinite dimensional system can be decomposed into an infinite number of mathematically tractable finite dimensional subsystems. The Laplace operator 
$\nabla^{2}$ can be written as

$$
\nabla^{2}=\mathcal{I}_{\Xi_{2}} \otimes \frac{\partial^{2}}{\partial \xi_{1}^{2}}+\frac{\partial^{2}}{\partial \xi_{2}^{2}} \otimes \mathcal{I}_{\Xi_{1}}
$$

with the tensor product $\otimes$ of the operators and the identity operators $\mathcal{I}_{\Xi_{i}}$ on $\Xi_{i}=\left[0, L_{i}\right](i=1,2)$. The eigenvalues $\lambda_{k, \ell}$ and the associated eigenfunctions $\phi_{k, \ell}(\boldsymbol{\xi})$ of $-\mu \nabla^{2}$ are

$$
\begin{aligned}
& \lambda_{k, \ell}:=\mu\left(\left(\frac{k \pi}{L_{1}}\right)^{2}+\left(\frac{\ell \pi}{L_{2}}\right)^{2}\right) \\
& \phi_{k, \ell}(\boldsymbol{\xi}):=\cos \left(\frac{k \pi \xi_{1}}{L_{1}}\right) \cos \left(\frac{\ell \pi \xi_{2}}{L_{2}}\right)
\end{aligned}
$$

with $k=0,1,2, \cdots$ and $\ell=0,1,2, \cdots$. This implies that we can diagonalize the Laplace operator by $(\mathcal{F} \otimes \mathcal{F}) \nabla^{2}\left(\mathcal{F}^{-1} \otimes \mathcal{F}^{-1}\right)$ with the Fourier transform operator $\mathcal{F}$. Since $\left(\mathcal{F}^{-1} \otimes \mathcal{F}^{-1}\right)\left(\mathcal{I}_{\Xi_{2}} \otimes h(s) \mathcal{I}_{\Xi_{1}}+h(s) \mathcal{I}_{\Xi_{2}} \otimes \mathcal{I}_{\Xi_{1}}\right)(\mathcal{F} \otimes \mathcal{F})=\mathcal{I}_{\Xi_{2}} \otimes h(s) \mathcal{I}_{\Xi_{1}}+$ $h(s) \mathcal{I}_{\Xi_{2}} \otimes \mathcal{I}_{\Xi_{1}}$, the feedback system in Fig. 2 (Left) can be decomposed into an infinite number of subsystems $\Sigma_{k, \ell}(k=0,1,2, \cdots, \ell=0,1,2, \cdots)$ depicted in Fig. 2 (Right). The readers are referred to, for example, Chapters 4 and 10 of 31] for the detailed derivation of the eigenvalues and the eigenfunctions.

This decomposition corresponds to the spatial modal decomposition of the molecular communication network system, which is essentially the method of the separation of variables (see 32 and Chapter 4 of [31]). Thus, the dynamics of each subsystem $\Sigma_{k, \ell}$ represent those of each spatial mode $\phi_{k, \ell}(\boldsymbol{\xi})$, and it follows that

$$
\tilde{\boldsymbol{x}}(\boldsymbol{\xi}, t)=\sum_{k=0}^{\infty} \sum_{\ell=0}^{\infty} \boldsymbol{w}_{k, \ell}(t) \phi_{k, \ell}(\boldsymbol{\xi}),
$$

where $\boldsymbol{w}_{k, \ell}$ is the state of the subsystem $\Sigma_{k, \ell}$.

This intuitively suggests that the concentrations perturbed around the homogeneous equilibrium $\overline{\boldsymbol{x}}$ eventually come back to $\overline{\boldsymbol{x}}$, when the growth rates of all the spatial modes are negative. On the other hand, if the growth rate of a non-zero spatial mode, $\phi_{k, \ell}(\xi)$ with $k+\ell \geq 1$, is positive, we expect the formation of a spatially periodic pattern of concentration gradient, or a Turing pattern. Therefore, the stability analysis of the molecular communication network is reduced to checking the stability of the finite-order subsystems $\Sigma_{k, \ell}$.

Remark 1. In general, the eigenvalues and the eigenfunctions defined in (9) and 10 can be written as

$$
\lambda_{\boldsymbol{k}}=\mu \sum_{i=1}^{N}\left(\frac{\lambda_{k_{i}} \pi}{L_{i}}\right)^{2}, \phi_{\boldsymbol{k}}(\boldsymbol{\xi})=\prod_{i=1}^{N} \cos \left(\frac{k_{i} \pi \xi_{i}}{L_{i}}\right)
$$

when the spatial domain is $N$ dimension and Neumann boundary condition is imposed, where $\boldsymbol{k}$ is the set of subindices $\left(k_{1}, k_{2}, \cdots, k_{N}\right)$ concerning the spatial 
dimension and $L_{i}$ is the length of the $i$-th dimension. The pair of eigenvalues and eigenfunctions depends on the boundary condition. For example, the eigenvalues for the Dirichlet boundary condition are given by 12 with $k+\ell \geq 1$. In other words, the zero eigenvalue $\lambda_{0,0}=0$ is not an eigenvalue for the Dirichlet condition. The associated eigenfunctions are $\phi_{\boldsymbol{k}}(\boldsymbol{\xi})=\prod_{i=1}^{N} \sin \left(k_{i} \pi \xi_{i} / L_{i}\right)$. We refer the readers to Chapters 10 and 11 of 31 ] for other boundary conditions. In the rest of this paper, we focus on the case of two dimensional spatial domain $\Omega$ with the Neumann boundary condition, since biomolecular communication networks would be implemented on a two dimensional surface.

\section{$3 \quad$ Stability analysis method for linearized model}

In this section, we provide a systematic local stability analysis tool for the linearized model (3) of the molecular communication networks using a control theoretic tool. The method is demonstrated on a novel activator-repressor-diffuser system. In particular, we show that the instability counterpart of the method is useful for the design and analysis of coordinated spatial pattern formation of the synthetic biocircuits.

\subsection{Graphical stability analysis method based on root lo- cus}

Suppose the internal circuit inside a cell is composed of $n \geq 2$ molecular species, and let the matrix $A \in \mathbb{R}^{n \times n}$ be partitioned as

$$
A=\left[\begin{array}{c:c}
A_{\text {circ }} & \boldsymbol{a}_{\mathrm{cd}} \\
\hdashline \boldsymbol{a}_{\mathrm{dc}}^{-} & a_{\mathrm{diff}}
\end{array}\right]
$$

with $A_{\text {circ }} \in \mathbb{R}^{(n-1) \times(n-1)}, \boldsymbol{a}_{\mathrm{cd}} \in \mathbb{R}^{n-1}, \boldsymbol{a}_{\mathrm{dc}} \in \mathbb{R}^{1 \times(n-1)}$ and $a_{\mathrm{diff}}<0$. Then, the matrix $A_{\text {circ }}$ represents the structure of the internal circuit, and $a_{\text {diff }}(<$ $0)$ is the degradation rate of the single diffusible molecule. The vectors $\boldsymbol{a}_{\mathrm{cd}}$ and $\boldsymbol{a}_{\mathrm{dc}}$ correspond to the interactions between the internal circuit molecules $\mathcal{M}_{1}, \mathcal{M}_{2}, \cdots, \mathcal{M}_{n-1}$ and the diffuser $\mathcal{M}_{n}$ (see Fig. 1). When $n=1$, we define $A=a_{\text {diff }}$.

Let a polynomial $p_{\lambda}(s)$ be defined by

$$
p_{\lambda}(s):=d(s)+\lambda n(s)
$$

with $d(s)$ and $n(s)$ in $(7)$. The characteristic polynomial of each subsystem $\Sigma_{k, \ell}$ is then written as $p_{\lambda_{k, \ell}}(s)=d(s)+\lambda_{k, \ell} n(s)$. The following lemma provides a physical interpretation of the poles and zeros of $h(s)$ using $A$ and $A_{\text {circ. }}$.

Lemma 1. Consider the molecular communication network system (3). Then, the characteristic polynomial $p_{\lambda}(s)$ of the system can be written as

$$
p_{\lambda}(s)=d(s)+\lambda n(s)=\left|s I_{n}-A\right|+\lambda\left|s I_{n-1}-A_{\text {circ }}\right|,
$$


where we define $\left|s I_{0}-A_{\text {circ }}\right|=1$ when $n=1$.

The proof can be found in Appendix. The lemma states that the zeros of $h(s)$ are determined from the dynamics of the internal circuit specified by $A_{\text {circ }}$, while the poles are determined from the overall reaction dynamics including the diffuser $\mathcal{M}_{n}$. Regarding Assumption 1, we note that $n(s)=\left|s I_{n-1}-A_{\text {circ }}\right|=0$ does not have roots on the imaginary axis for almost all $A_{\text {circ }}$ when all diagonal entries of $A_{\text {circ }}$ are non-zero. This is because the coefficient of the $s^{n-2}$ term is given by $\operatorname{Tr}\left(A_{\text {circ }}\right)$. In fact, the diagonal entries of $A_{\text {circ }}$ are non-zero in many biomolecular systems due to degradation and autoregulation.

It follows that the subsystem $\Sigma_{k, \ell}$ is asymptotically stable, if and only if all the roots of $p_{\lambda_{k, \ell}}(s)=0$ lie in the open left-half complex plane, also called Hurwitz. Since each subsystem represents the dynamics of each spatial mode as shown in the previous section, the stability of the molecular communication network (3) is guaranteed, if and only if all of the infinite number of finite dimensional subsystems $\Sigma_{k, \ell}(k=0,1,2, \cdots, \ell=0,1,2, \cdots)$ are stable.

Proposition 1. Suppose Assumption 1 holds. The molecular communication network (3) is exponentially stable if and only if $p_{\lambda_{k, \ell}}(s)$ is Hurwitz for all $k, \ell \in \mathbb{N}_{0}$.

Proof. We show the proof for the case of one-dimensional spatial domain in order to avoid notational clutter. However, generalization to multiple spatial dimensions is straightforward. Let $\left\{\sigma_{k, i}\right\}_{i=1}^{n}$ denote the roots of $p_{\lambda_{k}}(s)=0$. Then, it follows that

$$
\boldsymbol{w}_{k}(t)=\sum_{i=1}^{n} c_{k, i} e^{\sigma_{k, i} t} \boldsymbol{w}_{k}(0)
$$

where $c_{k, i}(i=1,2, \cdots, n)$ are some constant.

$(\Rightarrow)$ Suppose all of the polynomials $p_{\lambda_{k}}(s)=0\left(k \in \mathbb{N}_{0}\right)$ are Hurwitz. This means that there exists a positive real number $\epsilon_{1}$ such that $\operatorname{Re}\left[\sigma_{k, i}\right]<-\epsilon_{1}(<0)$ for all $k \in \mathbb{N}_{0}$. In the limit of $k \rightarrow \infty$, the eigenvalue $\lambda_{k} \rightarrow \infty$, and $n-1$ roots of $p_{\lambda_{k}}(s)$ converge to those of $n(s)=0$ and one root converges to $-\infty$, known as Butterworth pattern $[18$, since $n(s)$ is the $n-1$-th order polynomial as shown in Lemma 1 . Note that this rules out the possibility that the root asymptotically approaches to the imaginary axis. Let the roots of $n(s)=0$ be $\left\{\sigma_{\infty, i}\right\}_{i=1}^{n-1}$. Since $n(s)=0$ does not have roots on the imaginary axis from Assumption 1, there exists a positive real number $\epsilon_{2}$ such that $\operatorname{Re}\left[\sigma_{\infty, i}\right]<-\epsilon_{2}(<0)$ for all $i=1,2, \cdots, n-1$. Substituting (16) into (11) and taking the integral over the 
space $\Omega$ leads to

$$
\begin{aligned}
\int_{\Omega}\|\tilde{\boldsymbol{x}}(\xi, t)\| d \xi & =\int_{\Omega}\left\|\sum_{k=0}^{\infty}\left(\sum_{i=1}^{n} c_{k, i} e^{\sigma_{k, i} t} \boldsymbol{w}_{k}(0)\right) \phi_{k}(\xi)\right\| d \xi \\
& \leq C e^{-\epsilon t} \int_{\Omega}\left\|\sum_{k=0}^{\infty} \boldsymbol{w}_{k}(0) \phi_{k}(\xi)\right\| d \xi \\
& =C e^{-\epsilon t} \int_{\Omega}\|\tilde{\boldsymbol{x}}(\xi, 0)\| d \xi
\end{aligned}
$$

where $C$ is some constant and $\epsilon:=\min \left(\epsilon_{1}, \epsilon_{2}\right)$. Note that $\phi_{k}(\xi)=\cos \left(k \pi \xi / L_{1}\right)$ and the summation over $\ell$ in $(11)$ is dropped due to one dimensional spatial domain.

$(\Leftarrow)$ Suppose one of the polynomials $p_{\lambda_{k}}(s)=0$ is not Hurwitz, which implies the existence of $k_{0} \in \mathbb{N}_{0}$ and $i_{0} \in\{1,2, \cdots, n\}$ such that $\operatorname{Re}\left[\sigma_{k_{0}, i_{0}}\right] \geq 0$. This implies that the growth rate of the mode $\phi_{k_{0}}(\xi)$ is non-negative, and the right-hand side of the first equality of $(17)$ is bounded from below by $C e^{\operatorname{Re}\left[\sigma_{k_{0}, i_{0}}\right] t}\left\|\boldsymbol{w}_{k_{0}}(0)\right\|$ with some constant $C$.

Proposition 1 allows us to analyze the stability of the infinite dimensional system (3) using the finite dimensional subsystems $\Sigma_{k, \ell}(k=0,1,2, \cdots, \ell=$ $0,1,2, \cdots)$. It should be noted that there are still infinite subsystems $\Sigma_{k, \ell}(k=$ $0,1,2, \cdots, \ell=0,1,2, \cdots)$, although each subsystem is a finite dimensional system. In what follows, we show that the root locus method [18 allows us to systematically analyze the stability of the infinite number of subsystems $\Sigma_{k, \ell}$.

It follows that the roots of $p_{\lambda_{k, \ell}}(s)=0(k=0,1,2, \cdots, \ell=0,1,2, \cdots)$ lie on the root locus of the polynomial $p_{\lambda}(s)=d(s)+\lambda n(s)$, which is the characteristic polynomial of a feedback system composed of $h(s)$ and a feedback gain $\lambda$ (see Fig. 2 (Right)). Moreover, the definition (9) implies that the feedback gain $\lambda=\lambda_{k, \ell}$ monotonically increases in terms of $k$ and $\ell$. Thus, the root locus starts from the point where $\lambda=\lambda_{0,0}=0$ and converges to the point where $\lambda=\lim _{k, \ell \rightarrow \infty} \lambda_{k, \ell}$. This observation leads to the following graphical algorithm for stability analysis.

Algorithm 1. Given the dynamics of a single cell $h(s)$, we draw the root locus $\mathcal{R}$ of a negative feedback system composed of $h(s)$ and a constant feedback gain $\lambda$ with varying $\lambda \in[0, \infty]$. If the root locus $\mathcal{R}$ lies inside the open left-half complex plane $\mathbb{C}_{-}$, the molecular communication network $\sqrt{3}$ is stable.

This algorithm allows us to check the local stability of the molecular communication network system (3) using the transfer function of a single cell $h(s)$ and its root locus. Thus, the stability analysis of the infinite dimensional system is greatly simplified.

Remark 2. The reason that the root locus gives only a sufficient con- 
dition for stability is because the root locus is continuous in terms of the feedback gain $\lambda$, while the poles of the subsystems $\Sigma_{k, \ell}(k=0,1,2, \cdots, \ell=$ $0,1,2, \cdots)$ are discrete. In practice, however, the length of the spatial domain $L_{1}$ and $L_{2}$ is sufficiently large such that the discrete feedback gains $\lambda_{k, \ell}=$ $\mu\left(\left(k \pi / L_{1}\right)^{2}+\left(\ell \pi / L_{2}\right)^{2}\right) \quad(k=0,1,2, \cdots, \ell=0,1,2, \cdots)$ are close to each other as shown in Section 3.2 , and the above algorithm can be practically considered as necessary and sufficient.

It is worth noting that the root locus can be systematically drawn based on a set of known rules 18. Specifically, the locus $\mathcal{R}$ starts from the $n$ poles of $h(s)$, and $n-1$ roots converge to the zeros of $h(s)$ and one pole goes to $+\infty$ or $-\infty$, since the relative degree of $h(s)$ is one.

Lemma 2. The root locus $\mathcal{R}$ starts from $\operatorname{spec}(A)$, and $n-1$ roots converge to $\operatorname{spec}\left(A_{\text {circ }}\right)$.

This is a direct consequence of Lemma 1 and the properties of the root locus 18. This lemma implies that the start and the terminal point of the root locus $\mathcal{R}$ can be characterized by the dynamics of the entire circuit and the internal circuit, respectively. This intuition is helpful from a synthetic biology viewpoint to determine the structure of the internal biocircuit to guarantee the stability/instability of the system.

Remark 3. Arcak 22 showed algebraic conditions to guarantee the convergence of reaction-diffusion systems to spatially uniform solutions. When applied to our problem, Theorem 1 of 22 provides a sufficient condition for all the subsystems $\Sigma_{k, \ell}$ with $k+\ell \geq 1$ to be exponentially stable. Thus, the condition is sufficient for the stability of the spatially homogeneous equilibrium $\overline{\boldsymbol{x}}$, provided that $A$ is Hurwitz, or equivalently $\Sigma_{0,0}$ is stable. However, this sufficient condition is conservative compared to Proposition 1, which is necessary and sufficient, and Algorithm 1 of this paper, since it is based on constituting a common Lyapunov solution that verifies the stability of all of the subsystems $\Sigma_{k, \ell}$. On the other hand, theorems shown in 22] can be used to certify the spatially uniformity of non-stationary solutions such as oscillations, while Proposition 1 cannot.

\subsection{Example: activator-repressor-diffuser system}

We illustrate the graphical stability analysis method using a hypothetical biochemical network shown in Fig. 3. The system is composed of activatorrepressor internal circuit and a single diffuser molecule that allows cell-to-cell communication. In Fig. 3, $A, R$ and $D$ stand for activator, repressor and diffuser, respectively. In the context of synthetic biocircuit design, one can choose an $\mathrm{AraC}$ protein as an (inducible) activator, i.e., $A$ in Fig. 3 , and the araBAD operon as the corresponding promoter $\mathrm{P}_{A}$. For the repressor $R$, commonly used 


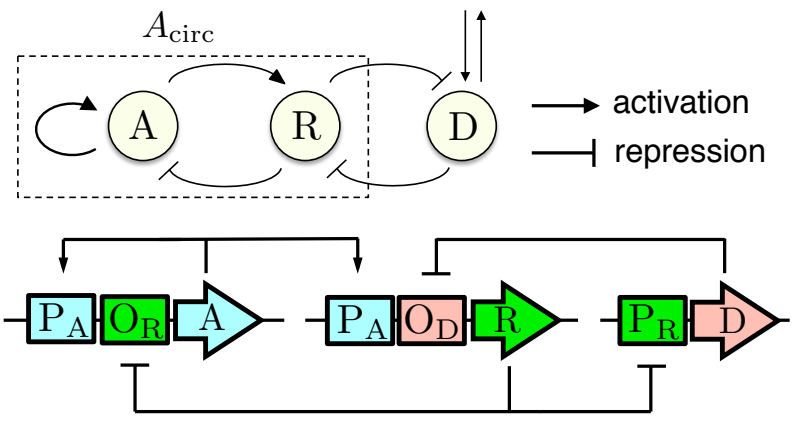

Figure 3: Schematic diagram of activator-repressor-diffuser system

TetR and LacI proteins and the corresponding promoters can be used. The diffuser is implemented with AHL, which is a bacterial quorum sensing molecule.

Let $a(\boldsymbol{\xi}, t), r(\boldsymbol{\xi}, t)$ and $d(\boldsymbol{\xi}, t)$ denote the concentration of activator, repressor and diffuser, respectively. The dynamics of the molecular communication network system are then modeled by

$$
\begin{aligned}
& \dot{a}=-\delta_{a} a+\gamma_{a} \frac{a^{2}}{K_{a}^{2}+a^{2}} \frac{K_{r}^{2}}{K_{r}^{2}+r^{2}} \\
& \dot{r}=-\delta_{r} r+\gamma_{r} \frac{a^{2}}{K_{a}^{2}+a^{2}} \frac{K_{d}^{2}}{K_{d}^{2}+d^{2}} \\
& \dot{d}=-\delta_{d} d+\gamma_{d} \frac{K_{r}^{2}}{K_{r}^{2}+r^{2}}+\mu \nabla^{2} d,
\end{aligned}
$$

where $\delta_{*}(*=a, r, d)$ and $\gamma_{*}(*=a, r, d)$ denote the degradation rates and the production rates of each molecular species, respectively.

Case A. (Stable case): Suppose the half-lives of activator, repressor and diffuser are 19.8 minutes, 55.5 minutes and 138.6 minutes, respectively, which equates to $\delta_{a}=0.035 \mathrm{~min}^{-1}, \delta_{r}=0.0125 \mathrm{~min}^{-1}$ and $\delta_{d}=0.0050 \mathrm{~min}^{-1}$. The production rates of each molecular species are $\gamma_{a}=2.5 \mu \mathrm{M} \cdot \min ^{-1}, \gamma_{r}=$ $1.65 \mu \mathrm{M} \cdot \min ^{-1}, \gamma_{d}=0.25 \mu \mathrm{M} \cdot \min ^{-1}$, and the Michaelis-Menten constants are $K_{a}=K_{r}=K_{d}=10 \mu \mathrm{M}$. The diffusion rate is $\mu=3.0 \times 10^{-4} \mathrm{~mm}^{2} \cdot \mathrm{min}^{-1}$. These parameter values are consistent with widely used parameters for numerical simulations in synthetic biology up to the order of magnitude (see $[9]$ for example). We leave the discussion on the realizability of such biomolecular communication networks in Remark 4 and focus on the numerical illustration of the root locus based analysis method here.

Substituting the parameters into the model $(18)-(20)$, we first calculate the spatially homogeneous equilibrium point of the system as $\left[a_{*}, r_{*}, d_{*}\right]^{T}=$ 
$[9.33,16.0,16.8]^{T}$. The Jacobian matrix $A$ around this equilibrium point is

$$
A=10^{-2} \times\left[\begin{array}{cc:c}
0.243 & -2.93 & 0 \\
2.29 & -1.25 & -1.76 \\
\hdashline 0 & -0 . \overline{7} 5 \overline{7} & -0 . \overline{5} 0 \overline{0}
\end{array}\right]
$$

The transfer function of a single cell $h(s)$ is then calculated from 15 as

$$
h(s)=\frac{s^{2}+1.01 \times 10^{-2} s+6.43 \times 10^{-4}}{s^{3}+1.51 \times 10^{-2} s^{2}+5.60 \times 10^{-4} s+3.54 \times 10^{-6}} .
$$

Figure 4 (Right) illustrates the root locus $\mathcal{R}$ drawn by Algorithm 1. The eigenvalues of $A$ and $A_{\text {circ }}$ are $\operatorname{spec}(A)=\{-0.00402 \pm 0.0221 j,-0.00702\}$ and $\operatorname{spec}\left(A_{\text {circ }}\right)=\{-0.00503 \pm 0.0248 j\}$, and we can see that these eigenvalues correspond to the initial and the terminal points of the root locus $\mathcal{R}$. Since the root locus $\mathcal{R}$ stays in the left-half complex plane, the equilibrium point of the molecular communication network is locally stable.

Figure 4 (Left) is the concentration gradient of the activator $a(\xi, t)$ at $t=$ $2860 \mathrm{~min}$. As expected from the graphical stability analysis result, the concentration converged to the spatially homogeneous equilibrium point. In the simulation, we used $L_{1}=3 \mathrm{~mm}$ and $L_{2}=2 \mathrm{~mm}$, and the initial values $a(\boldsymbol{\xi}, 0), r(\boldsymbol{\xi}, 0)$ and $d(\boldsymbol{\xi}, 0)$ were set as $x_{*}\left(1+0.01 \sum_{k=1}^{5} \sum_{\ell=1}^{5} \psi_{k, \ell}\left(\xi_{1}, \xi_{2}\right)\right)$, where $x_{*}$ is the equilibrium point of $a(\boldsymbol{\xi}, 0), r(\boldsymbol{\xi}, 0)$ and $(\boldsymbol{\xi}, 0)$, respectively, and

$$
\psi_{k, \ell}\left(\xi_{1}, \xi_{2}\right):=\cos \left(\frac{k \pi}{L_{1}}\left(\xi_{1}-\frac{L_{1}}{2}\right)\right) \cos \left(\frac{\ell \pi}{L_{2}}\left(\xi_{2}-\frac{L_{2}}{2}\right)\right) .
$$

The full temporal dynamics is available in a movie format [33], where the interval between frames is 5 minutes. We used COMSOL $\AA$ Multiphysics 4.4 for the simulation.

Case B. (Unstable case): Let $\gamma_{d}=0.30 \mu \mathrm{M} \cdot \mathrm{min}^{-1}$ and the rest of the parameters being kept the same as Case A. The equilibrium point is then $\left[a_{*}, r_{*}, d_{*}\right]^{T}=$ $[7.63,15.6,14.5]^{T}$. The eigenvalues of $A$ and $A_{\text {circ }}$ are calculated as $\operatorname{spec}(A)=$ $\{-0.000169 \pm 0.0237 j,-0.00792\}$ and $\operatorname{spec}\left(A_{\text {circ }}\right)=\{-0.00163 \pm 0.0258 j\}$. As shown in Fig. 5 (Right), the root locus lies in the open right-half complex plane. In fact, multiple roots of $p_{\lambda}(s)=0$ lie in the right-half complex plane, implying that the corresponding subsystems, or spatial modes, are unstable.

Figure 5 (Left) illustrates the concentration gradient of the activator $A$ at $t=2860 \mathrm{~min}$. Since the equilibrium point is not stable, the concentration does not converge to the spatially homogeneous equilibrium. In fact, a coordinated time-varying spatial pattern appeared in this example, which implies that the system fell into limit cycle instead of converging to a spatially inhomogeneous equilibrium point. The full temporal dynamics is available in a movie format [33, where the interval between frames is 5 minutes. We used COMSOL $\mathbb{R}$ Multiphysics 4.4 for the simulation. 

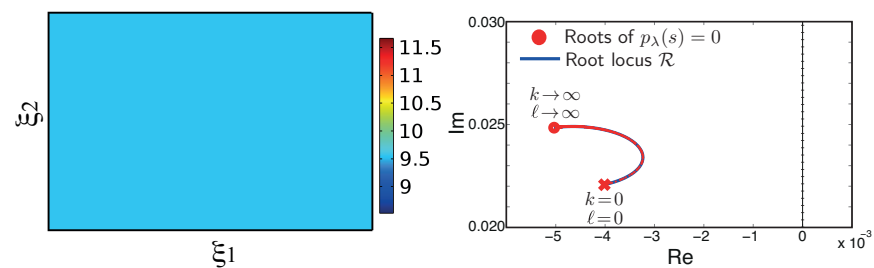

Figure 4: Case A: (Left) Concentration gradient of the activator $A$ over cell population at $t=2860 \mathrm{~min}$. (Right) Root locus $\mathcal{R}$ of the single cell dynamics $h(s)$.
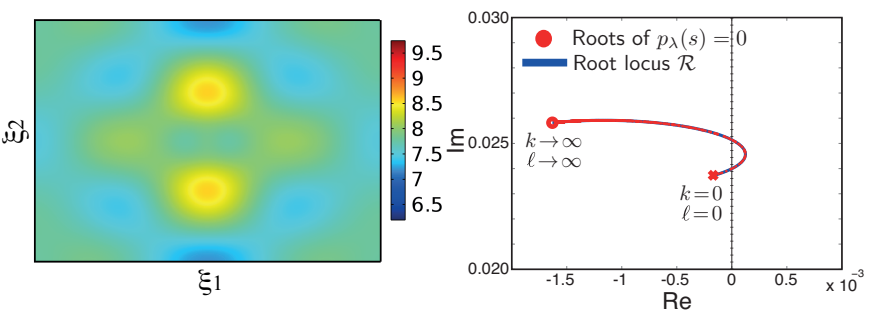

Figure 5: Case B: (Left) Concentration gradient of the activator $A$ over cell population at $t=2860 \mathrm{~min}$. (Right) Root locus $\mathcal{R}$ of the single cell dynamics $h(s)$.

As Turing [15] pointed out, this coordinated pattern formation can be explained by the fact that the subsystems corresponding to the non-zero spatial modes are destabilized. In the next section, we provide more rigorous argument of why and when the pattern formation can be expected.

Remark 4. The libraries of activators [34], repressors 35 and ribosome binding sites 36 recently became available for the use in synthetic biology. These libraries help us tune the rate parameters in a relatively predicted manner and would be useful for the implementation of the activator-repressor-diffuser biocircuit. In fact, it is encouraging that multiple biocircuits were implemented and tuned using these parts libraries $34,35,37]$. In the context of biomolecular communication network, the use of zinc finger proteins and small RNAs were also proposed in 38 .

\section{Conditions for Turing Pattern Formation}

\subsection{Finite mode Turing instability}

As seen in the previous example, molecular communication networks can exhibit coordinated spatial patterns based on the passive communication mechanism, 

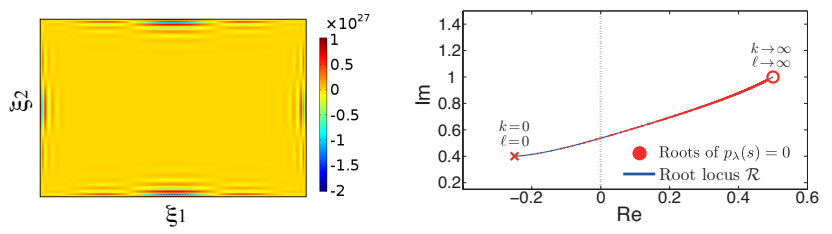

Figure 6: (Left) Concentration gradient of the activator $A$. (Right) Root locus $\mathcal{R}$ of the cell dynamics $h(s)$.

or diffusion. This is because non-zero finite spatial modes are destabilized and the growth rate of $\boldsymbol{w}_{k, \ell}$ in (11) is positive.

The instability of the non-zero spatial mode caused by diffusion is called Turing instability named after Alan Turing, who first pointed out the mathematical basis of the emergence of inhomogeneous spatial patterns in reaction-diffusion equations [15. In what follows, we introduce a formal definition of finite mode Turing instability for biomolecular communication networks, then we investigate conditions for the Turing instability.

Definition 1. (Finite mode Turing instability) Suppose Assumption 1 holds. A biomolecular communication network (1) is finite mode Turing unstable around an equilibrium $\overline{\boldsymbol{x}}$, if

(i) $p_{\lambda_{0,0}}(s)=p_{0}(s)=d(s) \neq 0 ; \forall s \in \mathbb{C}_{0+}$, and

(ii) $p_{\lambda}(\gamma+s)=0 ; \exists \lambda \in(0, \infty)$ and $\exists s \in \mathbb{C}_{+}$, where $\gamma=\max \left(\operatorname{Re}\left[s_{0}\right], 0\right)$ and $s_{0}$ is a root of $n(s)=0$ with the largest real part.

The condition (i) implies that the dynamics of each cell $h(s)$ is stable around an equilibrium point $\overline{\boldsymbol{x}}$ when there is no diffusion. The condition (ii) guarantees that the root locus of $h(s)$ goes into the open right-half plane of the complex plane for some feedback gain $\lambda>0$ (see Fig. 2 (Right)). In particular, the roots of $n(s)=0$ are the terminal points of the root locus (Lemma 2), thus the condition (ii) also implies that the right-most roots of $p_{\lambda}(s)=0$ are given when $\lambda$ is a non-zero and finite value. This guarantees that the dominant unstable spatial mode is finite and the resulting spatial pattern is of a non-zero finite spatial mode. We note that finite mode Turing instability is determined by the properties of the internal circuit $A_{\text {circ }}$ and $A$ but not the diffusion rate $\mu$ as shown in Definition 1. In practice, the wavelength of the dominant spatial mode needs to be longer than the size of a single cell. This can be tuned by varying the diffusion rate $\mu$ after the finite Turing instability is guaranteed by the biocircuit structures $A_{\text {circ }}$ and $A$.

It is important to guarantee that the dominant spatial mode is finite, since it is physically and biologically implausible to achieve coordinated spatial patterns of infinite spatial mode at steady state. The following toy example shows the case where the right-most roots of $p_{\lambda}(s)=0$ is given in the limit of $\lambda \rightarrow \infty$. 
Example: Suppose

$$
A=\left[\begin{array}{cc:c}
1.954 & -3.114 & 0 \\
1.0 & -0.9540 & -1.223 \\
\hdashline 0 & 1.0 & -2.0
\end{array}\right],
$$

and $\mu=0.006, L_{1}=3$ and $L_{2}=2$. We can calculate the dynamics of a single cell $h(s)$ using $A$ and the sub-matrix $A_{\text {circ }}$ and obtain the root locus $\mathcal{R}$ of $h(s)$. Figure 6 (Right) shows the right-most pole of the root locus $\mathcal{R}$. As $k, \ell \rightarrow \infty$, or equivalently $\lambda \rightarrow \infty$, the root of $p_{\lambda}(s)=0$ converges to the root of $n(s)=0$, or $s_{0}$. This implies that the growth rate of the state $\tilde{\boldsymbol{x}}$ is the largest for the infinite spatial mode, which is not plausible in real physical and biological systems. In fact, the simulation result in Fig. 6 (Left) shows spatial patterns with nonrealistic high spatial frequency. The time axis of the simulation, $\tau$, was scaled by $\tau=0.005 t$. The full temporal dynamics is available in a movie format 33 . We used COMSOL $\AA$ Multiphysics 4.4 for the simulation.

It should be noted that the importance of the finite mode instability was not actively discussed in many classical works [14, 39] where the systems are composed of $n=2$ molecular species and both can diffuse in the spatial domain. This is because the dominant spatial mode is always guaranteed to be finite mode, no matter how the reaction rates are varied.

\subsection{Condition for finite mode Turing instability}

In this section, we show that at least three molecular species, $\mathcal{M}_{1}, \mathcal{M}_{2}$ and $\mathcal{M}_{3}$, are necessary to achieve finite mode Turing instability in molecular communication networks. This implies that the well-known activator-repressor model [14 fails to make spatial patterns when there is only one diffusible molecule in the system.

Assumption 2. The matrix $A$ in the model (3) is Hurwitz.

This means that the reaction dynamics of a single cell $h(s)$ is stable. As Definition 1 shows, the stability of $h(s)$ is a necessary condition for finite mode Turing instability, and the existence of spatial patterns is essentially determined by the condition (ii) of Definition 1. Hence, by imposing Assumption 2, we hereafter focus on the condition (ii) of Definition 1.

The following lemma provides the necessary and sufficient condition for finite mode Turing instability.

Lemma 3. Consider the biomolecular communication network system (1) and its linearized system (3). Suppose Assumptions 1 and 2 hold. Then, the system (1) is finite mode Turing unstable around an equilibrium $\overline{\boldsymbol{x}}$, if and only if either of (a) or (b) holds. 
(a) $A_{\text {circ }}$ is Hurwitz, and $\operatorname{Re}\left[p_{\lambda}(j \omega)\right]=0, \operatorname{Im}\left[p_{\lambda}(j \omega)\right]=0$ for some $\lambda>0$ and some $\omega \in \mathbb{R}$.

(b) $A_{\text {circ }}$ is not Hurwitz, and $\operatorname{Re}\left[p_{\lambda}(\gamma+j \omega)\right]=0, \operatorname{Im}\left[p_{\lambda}(\gamma+j \omega)\right]=0$ for some $\lambda>0$ and some $\omega \in \mathbb{R}, \gamma:=\max _{\nu \in \operatorname{spec}\left(A_{\text {circ }}\right)} \operatorname{Re}[\nu]$

This lemma is a direct consequence of Definition 1. The conditions (a) and (b) are equivalent to (ii) of Definition 1 with $\gamma=\operatorname{Re}\left[s_{0}\right]$ and $\gamma=0$, respectively.

Using this lemma, we can derive the following theorem showing that at least three molecular species are necessary to achieve finite mode Turing instability.

Theorem 1. Consider the biomolecular communication network system (1) and its linearized system (3). Suppose Assumptions 1 and 2 hold. Then, the system (1) composed of $n=1$ and $n=2$ molecular species cannot be finite mode Turing unstable.

The proof of this theorem can be found in Appendix. This theorem implies that the activator-repressor-diffuser system illustrated in Section 3.2 is one of the minimal biomolecular communication networks that can exhibit finite mode Turing instability with $n=3$.

Moreover, the following theorem shows that finite Turing instability is always caused by Hopf-Turing bifurcation, implying that not only coordinated spatial patterns but also temporal oscillations of concentration gradients are expected at steady state.

Theorem 2. Consider the biomolecular communication network system (1) and its linearized system (3). Suppose the system (1) is finite mode Turing unstable around an equilibrium $\overline{\boldsymbol{x}}$. Then, the right-most pole of the characteristic polynomial $p_{\lambda}(s)=0$ is a pair of complex conjugate.

Proof. We prove by contradiction. Suppose the right-most root of $p_{\lambda}(s)=0$ is a real number $\sigma$, and it is given when $\lambda=\lambda_{0}$. That is, $p_{\lambda_{0}}(\sigma)=0$. It follows from (i) of Definition 1 that all of the poles of $h(s)$ lie in $\mathbb{C}_{-}$. Moreover, (ii) of Definition 1 implies that $\sigma>\max \left(\operatorname{Re}\left[s_{0}\right], 0\right) \geq 0$, where $s_{0}$ is a root of $n(s)=0$ with the largest real part. Therefore, $\sigma$ is greater than any real poles and zeros of $h(s)$. This, however, contradicts with the property of the root locus that the locus exists on real axis to the left of an odd number of poles and zeros of $h(s)$ [18].

In the next section, we turn our attention to the synthesis of the minimal molecular communication networks with $n=3$ molecular species and investigate their properties in detail. 


\section{APPLICATION TO THREE-COMPONENT CIRCUIT SYNTHESIS}

In this section, we consider molecular communication networks consisting of two internal circuit molecules, $\mathcal{M}_{1}$ and $\mathcal{M}_{2}$, and one diffuser $\mathcal{M}_{3}$. For this class of systems, we first derive an analytic condition for finite mode Turing instability. Using the analytic condition, we characterize a structural property of biomolecular communication networks that admit Turing instability.

Let $\alpha_{i}$ and $\beta_{i}$ denote the coefficients of the following characteristic polynomials.

$$
\begin{aligned}
& \left|s I_{3}-A\right|=s^{3}+\alpha_{2} s^{2}+\alpha_{1} s+\alpha_{0}, \\
& \left|s I_{2}-A_{\text {circ }}\right|=s^{2}+\beta_{1} s+\beta_{0} .
\end{aligned}
$$

Then, a necessary and sufficient condition for finite mode Turing instability is given by the following theorem.

Theorem 3. Consider the biomolecular communication network system (1) composed of $n=3$ molecular species and its linearized system (3). Suppose Assumptions 1 and 2. The system (1) is finite mode Turing unstable around an equilibrium $\overline{\boldsymbol{x}}$, if and only if the following $(A)$ or (B) is satisfied.

$$
\begin{array}{ll}
\text { (A) } & \beta_{1}>0, \beta_{0}>0, \\
& \alpha_{1}+\beta_{1} \alpha_{2}-\beta_{0} \leq-2 \sqrt{\beta_{1}\left(\alpha_{1} \alpha_{2}-\alpha_{0}\right)}, \\
\text { (B) } & \beta_{1}<0, \beta_{1}^{2}-4 \beta_{0}<0, \\
& -\beta_{1}^{2}+\beta_{0}+\beta_{1} \alpha_{2}-\alpha_{1}>0 .
\end{array}
$$

The conditions (A) and (B) in Theorem 3 are equivalent to (a) and (b) of Lemma 3, respectively. The complete proof is shown in Appendix. It should be noticed that $\beta_{i}(i=0,1)$ are determined from the parameters of the internal circuit $A_{\text {circ }}$, while $\alpha_{i}(i=0,1,2)$ depend on the entire system including the diffuser. Theorem 3 allows us to further investigate the properties of threecomponent systems. In what follows, we show that a molecular communication network needs to have a certain structural property to admit finite mode Turing instability.

We consider a class of cascaded three-component circuits illustrated in Fig. 7. The matrix $A$ is then defined by

$$
A=\left[\begin{array}{cc:c}
a_{11} & a_{12} & 0 \\
a_{21} & a_{22} & a_{23} \\
\hdashline 0 & a_{32} & a_{\text {diff }}
\end{array}\right] \text {. }
$$

The activator-repressor-diffuser network in Section 3.2 is a class of the cascaded circuit with $a_{12}<0, a_{21}>0, a_{23}<0$ and $a_{32}<0$. Note that the signs 


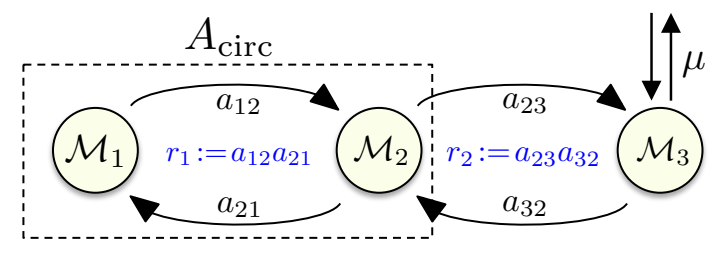

Figure 7: Schematic diagram of three-component circuit

of $a_{12}, a_{21}, a_{23}$ and $a_{32}$ depend on whether the molecule $\mathcal{M}_{1}, \mathcal{M}_{2}$ and $\mathcal{M}_{3}$ are activator or repressor. The following proposition specifies the combinations of activator and repressors that admit finite mode Turing instability.

Proposition 2. Consider the biomolecular communication networks with the cascaded structure shown in Fig. 7. Suppose Assumptions 1 and 2 hold. Let $r_{1}:=a_{12} a_{21}$ and $r_{2}:=a_{23} a_{32}$. Then, the network admits finite mode Turing instability only if $r_{1}<0$ and $r_{2}>0$.

The proof can be found in Appendix. This proposition narrows down the design space of biomolecular communication networks. In other words, the combination of the molecules $\mathcal{M}_{1}, \mathcal{M}_{2}$ and $\mathcal{M}_{3}$ needs to be either of (activator, repressor, repressor) or (repressor, activator, activator) in order for the system to achieve finite mode Turing instability. It should be noticed that the example shown in Section 3.2 is an example of (activator, repressor, repressor) configuration.

\section{Conclusion}

In this paper, we have proposed a control theoretic modeling and analysis framework for biomolecular communication networks modeled by reaction-diffusion equations with a single diffusible molecule. We have first shown that the analysis of the infinite dimensional system can be simplified by decomposing it into finite dimensional subsystems. We have then provided a graphical stability analysis tool using the root locus approach and demonstrated on the novel activator-repressor-diffuser system, which allows for the formation of inhomogeneous concentration gradient over the cell population. The latter half of the paper has been devoted to analyzing the conditions for finite mode Turing instability. Using the analytic condition of Turing instability, we have shown that the network needs to have at least three molecular species to admit Turing instability. Moreover, the in-depth study of three-component circuits provided a necessary activation-repression network structure condition for Turing instability. Our future work will be devoted to addressing robustness issues such as cell-to-cell variability and parameter uncertainties of biomolecular communication networks. The activator-repressor-diffuser system will also need further 
study to characterize the parameter regions for Turing instability.

\section{References}

[1] I. F. Akyildiz, F. Fekri, R. Sivakumar, C. R. Forest, and B. K. Hammer, "MONACO: Fundamentals of molecular nano-communication networks," IEEE Wireless Communications, vol. 19, no. 5, pp. 12-18, 2012.

[2] T. Nakano, T. Suda, Y. Okaie, M. J. Moore, and A. V. Vasilakos, "Molecular communication among biological nanomachines: a layered architecture and research issue," IEEE Transactions on NanoBioscience, vol. 13, no. 3, pp. 169-197, 2014.

[3] T. Danino, O. Mondragón-Palomino, L. Tsimring, and J. Hasty, "A synchronized quorum of genetic clocks," Nature, vol. 463, no. 7279, pp. 326$330,2010$.

[4] L. You, R. S. C. III, R. Weiss, and F. H. Arnold, "Programmed population control by cell-cell communication and regulated killing," Nature, vol. 428, no. 6985 , pp. 868-871, 2004.

[5] F. K. Balagaddé, H. Song, J. Ozaki, C. H. Collins, M. Barnet, F. H. Arnold, S. R. Quake, and L. You, "A synthetic Escherichia coli predatorprey ecosystem," Molecular Systems Biology, vol. 4, no. 1, 2008.

[6] J. J. Tabor, H. Salis, Z. B. Simpson, A. A. Chevalier, A. Levskaya, E. M. Marcotte, C. A. Voigt, and A. D. Ellington, "A synthetic genetic edge detection program," Cell, vol. 137, no. 7, pp. 1272-1281, 2009.

[7] A. Tamsir, J. J. Tabor, and C. A. Voigt, "Robust multicellular computing using genetically encoded nor gates and chemical wires," Nature, vol. 469, no. 7329 , pp. 212-215, 2011.

[8] S. Regot, J. Macia, N. Conde, K. Furukawa, J. Kjellén, T. Peeters, S. Hohmann, E. de Nadal, F. Posas, and R. Solé, "Distributed biological computation with multicellular engineered networks," Nature, vol. 469, no. 7329 , pp. 207-211, 2011.

[9] S. Basu, Y. Gerchman, C. H. Collins, F. H. Arnold, and R. Weiss, "A synthetic multicellular system for programmed pattern formation," Nature, vol. 434, no. 7037, pp. 1130-1134, 2005.

[10] C. Liu, X. Fu, L. Liu, X. Ren, C. K. Chau, S. Li, L. Xiang, H. Zeng, G. Chen, L.-H. Tang, P. Lenz, X. Cui, W. Huang, T. Hwa, and J.-D. Huang, "Sequential establishment of stripe patterns in an expanding cell population," Science, vol. 334, no. 6053, pp. 238-241, 2011. 
[11] A. Koch and H. Meinhardt, "Biological pattern formation: from basic mechanisms to complex structures," Reviews of Modern Physics, vol. 66, no. 4, pp. 1481-1507, 1994.

[12] S. Kondo and T. Miura, "Reaction-diffusion model as a framework for understanding biological pattern formation," Science, vol. 329, no. 5999, pp. 1616-1620, 2010.

[13] J. Hsia, W. J. Holtz, D. C. Huang, M. Arcak, and M. M. Maharbiz, "A feedback quenched oscillator produces Turing patterning with one diffuser," PLOS Computational Biology, vol. 8, no. 1, 2012, e1002331.

[14] L. Edelstein-Keshet, Mathematical models in biology. SIAM, 2005.

[15] A. M. Turing, "The chemical basis of morphogenesis," Philosophicals Transactions of the Royal Society of London B, vol. 237, no. 641, pp. 37-72, 1952.

[16] A. Gierer and H. Meinhardt, "A theory of biological pattern formation," Kybernetik, vol. 12, no. 1, pp. 30-39, 1972.

[17] H. G. Othmer, K. Painter, D. Umulis, and C. Xue, "The intersection of theory and application in elucidating pattern formation in developmental biology," Mathematical Modelling of Natural Phenomena, vol. 4, no. 4, pp. $3-82,2009$.

[18] W. S. Levine, The Control Handbook. CRC Press, 1996.

[19] H. Miyazako, Y. Hori, and S. Hara, "Turing instability in reaction-diffusion systems with a single diffuser: characterization based on root locus," in Proceedings of IEEE Conference on Decision and Control, 2013, pp. 26712676.

[20] Y. Hori, S. Kumagai, and S. Hara, "Network structure for Turing instabilizability in reaction-diffusion systems with one diffuser: a case study for three-gene networks," in Proceedings of SICE Annual Conference, 2014, pp. 892-895.

[21] H. Miyazako, Y. Hori, and S. Hara, "The analysis of Turing instability in reaction-diffusion systems using a single diffuser," Transactions of the Society of Instrument and Control Engineers, vol. 49, no. 12, pp. 1164-1171, 2013, (in Japanese).

[22] M. Arcak, "Certifying spatially uniform behavior in reaction-diffusion pde and compartmental ode systems," Automatica, vol. 47, no. 6, pp. 12191229, 2011.

[23] T. S. Gardner, C. R. Cantor, and J. J. Collins, "Construction of a genetic toggle switch in escherichia coli," Nature, vol. 403, no. 6767, pp. 339-342, 2000 . 
[24] M. B. Elowitz and S. Leibler, "A synthetic oscillatory network of transcriptional regulators," Nature, vol. 403, no. 6767, pp. 335-338, 2000.

[25] T.-S. Moon, C. Lou, A. Tamsir, B. C. Stanton, and C. A. Voigt, "Genetic programs constructed from layered logic gates in single cells," Nature, vol. 491, no. 7423, pp. 249-253, 2012.

[26] J. Morgan, "Global existence for semilinear parabolic systems," SIAM Journal on Mathematical Analysis, vol. 20, no. 5, pp. 1128-1144, 1989.

[27] J. Smoller, Shock waves and reaction-diffusion equations. Springer-Verlag, 1994.

[28] S. Hara, T. Hayakawa, and H. Sugata, "LTI systems with generalized frequency variables: A unified framework for homogeneous multi-agent dynamical systems," SICE Journal of Control, Measurement and System Integration, vol. 2, no. 5, pp. 299-306, 2009.

[29] S. Hara, H. Tanaka, and T. Iwasaki, "Stability analysis of systems with generalized frequency variables," IEEE Transactions on Automatic Control, vol. 59, no. 2, pp. 313-326, 2014.

[30] J. P. Hespanha, Linear systems theory. Princeton University Press, 2009.

[31] W. A. Strauss, Partial differential equations: An introduction, 2nd ed. John Wiley, 2008.

[32] R. G. Casten and C. J. Holland, "Instability results for reaction diffusion equations with neumann boundary conditions," Journal of Differential Equations, vol. 27, no. 2, pp. 266-273, 1978.

[33] Y. Hori, H. Miyazako, S. Kumagai, and S. Hara, "Coordinated spatial pattern formation in biomolecular communication networks," 2015, arXiv:1504.06045v3 (available at http://arxiv.org/abs/1504.06045).

[34] V. A. Rhodius, T. H. Segall-Shapiro, B. D. Sharon, A. Ghodasara, E. Orlova, H. Tabakh, D. H. Burkhardt, K. Clancy, T. C. Peterson, C. A. Gross, and C. A. Voigt, "Design of orthogonal genetic switches based on a crosstalk map of $\sigma \mathrm{s}$, anti- $\sigma \mathrm{s}$, and promoters," Molecular Systems Biology, vol. 9, p. 702, 2013.

[35] B. C. Stanton, A. A. K. Nielsen, A. Tamsir, K. Clancy, T. Peterson, and C. A. Voigt, "Genomic mining of prokaryotic repressors for orthogonal logic gates," Nature Chemical Biology, vol. 10, pp. 99-105, 2014.

[36] V. K. Mutalik, J. C. Guimaraes, G. Cambray, C. Lam, M. J. Christoffersen, Q.-A. Mai, A. B. Tran, M. Paull, J. D. Keasling, A. P. Arkin, and D. Endy, "Precise and reliable gene expression via standard transcription and translation initiation elements," Nature Methods, vol. 10, pp. 354-360, 2013. 
[37] H. Niederholtmeyer, Z. Sun, Y. Hori, E. Yeung, A. Verpoorte, R. M. Murray, and S. J. Maerkl, "A cell-free framework for biological systems engineering," bioRxiv, 2015, doi:10.1101/018317.

[38] J. Hsia, W. J. Holtz, M. M. Maharbiz, and M. Arcak, "New architecture for patterning gene expression using zinc finger proteins and small RNAs," in Proceedings of IEEE Conference on Decision and Control, 2012, pp. 1633-1638.

[39] J. Murray, Mathematical Biology II, 3rd ed. Springer, 2003.

\section{.1 Proof of Lemma 1}

Using the definition (7), we can write $h(s)$ as

$$
h(s)=\frac{\boldsymbol{e}_{n}{ }^{T} \operatorname{adj}\left(s I_{n}-A\right) \boldsymbol{e}_{n}}{\left|s I_{n}-A\right|},
$$

where $\operatorname{adj}(X)$ denotes the adjugate matrix of $X$. It follows from the definition of the adjugate matrix that the $(n, n)$-th entry of $\operatorname{adj}\left(s I_{n}-A\right)$ is $\left|s I_{n-1}-A_{\text {circ }}\right|$, and thus the numerator of $h(s)$ can be written as $\boldsymbol{e}_{n}^{T} \operatorname{adj}\left(s I_{n}-A\right) \boldsymbol{e}_{n}=\mid s I_{n-1}-$ $A_{\text {circ }} \mid$.

\section{$.2 \quad$ Proof of Theorem 1}

For $n=1$, it follows that the molecular communication network is finite mode Turing unstable if and only if the characteristic polynomial $p_{\lambda}(s)=s-a_{\text {diff }}+\lambda$ satisfies (a) of Lemma 3 . We can easily see that $\operatorname{Re}\left[p_{\lambda}(j \omega)\right]=0$ cannot be satisfied for all $\lambda>0$ and $\omega \in \mathbb{R}$, since $a_{\text {diff }}<0$.

For $n=2$, we define coefficient $\alpha_{0}, \alpha_{1}$ and $\beta_{0}$ by $\left|s I_{2}-A\right|=s^{2}+\alpha_{1} s+\alpha_{0}$ and $\left|s I_{2}-A_{\text {circ }}\right|=s+\beta_{0}$. In what follows, we show that both (a) and (b) of Lemma 3 cannot be satisfied.

Case 1: $A_{\text {circ }}$ is Hurwitz: It follows that $\operatorname{Im}\left[p_{\lambda}(j \omega)\right]=\omega\left(\alpha_{1}+\lambda\right)$ for all $\lambda>0$ and $\omega \neq 0$, since $\alpha_{1}>0$ holds when $A$ is Hurwtiz. For $\omega=0, \operatorname{Re}\left[p_{\lambda}(0)\right]=$ $\alpha_{0}+\lambda \beta_{0} \neq 0$ holds for all $\lambda>0$ because of $\alpha_{0}>0$ and $\beta_{0}>0$. Thus, the condition (a) in Lemma 3 cannot be satisfied.

Case 2: $A_{\text {circ }}$ is not Hurwitz: It follows that $\operatorname{Im}\left[p_{\lambda}(\gamma+j \omega)\right]=\omega\left(2 \gamma+\alpha_{1}+\lambda\right)$, where $\gamma=\beta_{0}(\geq 0)$ from the definition. Thus, $\operatorname{Im}[(\lambda, \gamma+j \omega)] \neq 0$ for all $\lambda>0$ and $\omega \neq 0$, since $\alpha_{1}>0$ and $\gamma \geq 0$. When $\omega=0, \operatorname{Re}\left[p_{\lambda}(\gamma)\right]=$ $\gamma^{2}+\gamma\left(\alpha_{1}+\lambda\right)+\alpha_{0}+\lambda \beta_{0}>0$ hold, and $\operatorname{Re}\left[p_{\lambda}(\gamma)\right] \neq 0$ hold for all $\lambda>0$. This completes the proof.

\section{$.3 \quad$ Proof of Theorem 3}

We prove the theorem by calculating conditions (a) and (b) of Lemma 3 for $n=3$. 
In what follows, we derive the conditions (A) and (B) by considering the case where $A_{\text {circ }}$ is Hurwitz and not Hurwitz, respectively. We can easily verify that neither (a) nor (b) of Lemma 3 can be satisfied when $\omega=0$. Hence, we hereafter show the proof for the case of $\omega \neq 0$.

Case 1: $A_{\text {circ }}$ is Hurwitz: A necessary condition for $A_{\text {circ }}$ being Hurwitz is

$$
\beta_{1}>0 \text { and } \beta_{0}>0 .
$$

Moreover, $p_{\lambda}(j \omega)=0$ implies that

$$
\begin{aligned}
& \operatorname{Re}\left[p_{\lambda}(j \omega)\right]=-\alpha_{2} \omega^{2}+\alpha_{0}+\lambda\left(-\omega^{2}+\beta_{0}\right)=0 \\
& \operatorname{Im}\left[p_{\lambda}(j \omega)\right]=-\omega\left\{\omega^{2}-\left(\alpha_{1}+\lambda \beta_{1}\right)\right\}=0 .
\end{aligned}
$$

It follows from 29 that $\omega^{2}=\alpha_{1}+\lambda \beta_{1}(>0)$, since $\omega \neq 0$. We can then eliminate $\omega^{2}$ from [28), and we have the following quadratic equation of $\lambda$.

$$
\beta_{1} \lambda^{2}+\left(\alpha_{1}+\beta_{1} \alpha_{2}-\beta_{0}\right) \lambda+\alpha_{1} \alpha_{2}-\alpha_{0}=0 .
$$

Since $\beta_{1}>0$ holds from (27), and $\alpha_{1} \alpha_{2}-\alpha_{0}>0$ holds from Assumption 2, a necessary and sufficient condition for $(30)$ having a positive real solution is given by the following $(\mathrm{C} 1)$ and $(\mathrm{C} 2)$.

(C1) The determinant of (30) is non-negative. That is,

$$
\left(\left(\alpha_{1}+\beta_{1}\right) \alpha_{2}-\beta_{0}^{2}\right)^{2}-4 \beta_{1}\left(\alpha_{1} \alpha_{2}-\alpha_{0}\right) \geq 0 .
$$

(C2) The coefficient of $\lambda$ in (30) is negative. That is,

$$
\alpha_{1}+\beta_{1} \alpha_{2}-\beta_{0}<0 \text {. }
$$

Summarizing (31) and (32), we have

$$
\alpha_{1}+\beta_{1} \alpha_{2}-\beta_{0} \leq-2 \sqrt{\beta_{1}\left(\alpha_{1} \alpha_{2}-\alpha_{0}\right)} .
$$

The condition (A) is obtained from (27) and (33).

Case 2: $A_{\text {circ }}$ is not Hurwitz A necessary condition for $A_{\text {circ }}$ not being Hurwitz is

$$
\beta_{1} \leq 0 \text { or } \beta_{0} \leq 0 .
$$

Let $\gamma \pm j \eta(\gamma, \eta \geq 0)$ denote a pair of eigenvalues of $A_{\text {circ }}$ with the largest real part. Then, $\left|(\gamma+j \eta) I_{2}-A_{\text {circ }}\right|=0$ implies

$$
\begin{aligned}
& \gamma^{2}+\beta_{1} \gamma+\beta_{0}-\eta^{2}=0 \\
& \left(2 \gamma+\beta_{1}\right) \eta=0 .
\end{aligned}
$$


In what follows, we first show that $p_{\lambda}(\gamma+j \eta) \neq 0$ for all $\lambda>0$ and $\omega$ when $\eta=0$. It follows from $\operatorname{Im}\left[p_{\lambda}(\gamma+j \omega)\right]=0$ that

$$
\omega^{2}=3 \gamma^{2}+2 \alpha_{2} \gamma+\alpha_{1}+\lambda\left(2 \gamma+\beta_{1}\right) .
$$

Substituting this into $\operatorname{Re}\left[p_{\lambda}(\gamma+j \omega)\right]=0$ yields the following equation of $\lambda$.

$$
\begin{aligned}
& \left(2 \gamma+\beta_{1}\right) \lambda^{2}+\left\{9 \gamma^{2}+\left(4 \alpha_{2}+3 \beta_{1}\right) \gamma+\alpha_{1}+\beta_{1} \alpha_{2}\right\} \lambda \\
& \quad+\left\{8 \gamma^{3}+8 \alpha_{2} \gamma^{2}+2\left(\alpha_{1}+\alpha_{2}^{2}\right) \gamma+\left(\alpha_{1} \alpha_{2}-\alpha_{0}\right)\right\}=0 .
\end{aligned}
$$

It should be noted that the coefficient of $\lambda^{2}$ is non-negative, or $2 \gamma+\beta_{1} \geq 0$, since $\gamma$ is the largest real root of $s^{2}+\beta_{1} s+\beta_{0}=0$ from the definition. Moreover, the coefficients of $\lambda$ and the constant terms of (38) are also positive because $\alpha_{0}>0, \alpha_{2}>0$ and $\alpha_{1} \alpha_{2}-\alpha_{0}>0$ from Assumption 2, and (34) holds. Thus, $p_{\lambda}(\gamma+j \eta) \neq 0$ for all $\lambda>0$ and $\omega$ when $\eta=0$.

When $\eta \neq 0$, the determinant of $\left|s I_{2}-A_{\text {circ }}\right|=0$ is negative, thus we have

$$
\beta_{1}^{2}-4 \beta_{0}<0
$$

Moreover, $\operatorname{Im}\left[p_{\lambda}(\gamma+j \omega)\right]=0$ implies

$$
\omega^{2}=3 \gamma^{2}+2 \alpha_{2} \beta+\alpha_{1} .
$$

Substituting this into $\operatorname{Re}\left[p_{\lambda}(\gamma+j \omega)\right]=0$ yields

$$
\begin{gathered}
\left\{\gamma^{2}-\left(3 \beta^{2}+2 \alpha_{2} \beta+\alpha_{1}\right)\right\} \lambda-\left\{8 \gamma^{3}+8 \alpha_{2} \gamma^{2}+\right. \\
\left.2\left(\alpha_{1}+\alpha_{2}^{2}\right) \gamma+\left(\alpha_{1} \alpha_{2}-\alpha_{0}\right)\right\}=0 .
\end{gathered}
$$

Note that the constant term of (41) is negative because of Assumption 2 and $\gamma>0$. Thus, 410 has a positive real root if and only if

$$
\eta^{2}-\left(3 \gamma^{2}+2 \alpha_{2} \gamma+\alpha_{1}\right)>0 .
$$

The condition (B) is obtained from (34), 335, 36, 39, and 42,.

\section{$.4 \quad$ Proof of Proposition 2}

We first show $r_{1}<0$. Suppose (A) of Theorem 3 holds. It follows from $\beta_{1}=$ $-\left(a_{11}+a_{22}\right)>0$ of $(\mathrm{A})$ that either or both of $a_{11}$ and $a_{22}$ need to be negative. It follows that $r_{1}=a_{12} a_{21}<0$ when $a_{11}$ and $a_{22}$ have opposite signs, hence we consider the case of $a_{11}<0$ and $a_{22}<0$ in the following.

We first note that $\alpha_{1} \alpha_{2}-\alpha_{0}>0$ holds from Assumption 2, and it can be equivalently written as

$$
\begin{aligned}
& \left(a_{11}+a_{22}\right) r_{1}+\left(a_{22}+a_{\text {diff }}\right) r_{2} \\
& \quad-\left(a_{11}+a_{22}\right)\left(a_{22}+a_{\text {diff }}\right)\left(a_{\text {diff }}+a_{11}\right)>0 .
\end{aligned}
$$


Dividing (43) by $a_{11}+a_{22}(<0)$, we have

$$
r_{1}<\left(a_{22}+a_{\text {diff }}\right)\left(a_{\text {diff }}+a_{11}\right)-\frac{a_{22}+a_{\text {diff }}}{a_{11}+a_{22}} r_{2} .
$$

On the other hand, $r_{2}$ satisfies the following inequality from $\alpha_{1}+\beta_{1} \alpha_{2}-\beta_{0} \leq$ $-2 \sqrt{\beta_{1}\left(\alpha_{1} \alpha_{2}-\alpha_{0}\right)} \leq 0$ of $(\mathrm{A})$.

$$
\left(a_{11}+a_{22}\right)\left(a_{11}+a_{22}+2 a_{\text {diff }}\right)-r_{2} \leq 0 .
$$

Since $a_{11}<0, a_{22}<0$ and $a_{\text {diff }}<0$, the second term on the right side of (44) satisfies

$$
-\frac{a_{22}+a_{\text {diff }}}{a_{11}+a_{22}} r_{2}<-\left(a_{22}+a_{\text {diff }}\right)\left(a_{11}+a_{22}+2 a_{\text {diff }}\right) .
$$

Summerizing (44) and (46), we have

$$
\begin{aligned}
r_{1}< & \left(a_{22}+a_{\text {diff }}\right)\left(a_{\text {diff }}+a_{11}\right)-\frac{a_{22}+a_{\text {diff }}}{a_{11}+a_{22}} r_{2} \\
< & \left(a_{22}+a_{\text {diff }}\right)\left(a_{\text {diff }}+a_{11}\right) \\
& \quad-\left(a_{22}+a_{\text {diff }}\right)\left(a_{11}+a_{22}+2 a_{\text {diff }}\right) \\
= & -\left(a_{22}+a_{\text {diff }}\right)^{2}<0 .
\end{aligned}
$$

Suppose (II-B) of Theorem 3 holds. It then follows from $\beta_{1}^{2}-4 \beta_{0}<0$ of (B) that

$$
\left(a_{11}+a_{22}\right)^{2}-4\left(a_{11} a_{22}-r_{1}\right)=\left(a_{11}-a_{22}\right)^{2}+4 r_{1}<0 .
$$

This concludes $r_{1}<0$.

Next, we show that $r_{2}>0$ is necessary. Suppose (B) of Theorem 3 holds. Then, the direct calculation leads to $-\beta_{1}^{2}+\beta_{0}+\beta_{1} \alpha_{2}-\alpha_{1}=r_{2}>0$. In what follows, we consider the case where (A) of Theorem 3 holds.

It follows from $a_{\text {diff }}<0$ and $\beta_{1}=-\left(a_{1}+a_{2}\right)>0$ of (A) that $\left(a_{1}+a_{2}+2 a_{3}\right)<$ 0 . Then, $\alpha_{1}+\beta_{1} \alpha_{2}-\beta_{0} \leq-2 \sqrt{\beta_{1}\left(\alpha_{1} \alpha_{2}-\alpha_{0}\right)} \leq 0$ of (A) implies (45). Thus, $r_{2}>\left(a_{1}+a_{2}\right)\left(a_{1}+a_{2}+2 a_{3}\right)>0$. 